\section{Laser kann topische Therapie von Nagelpilz unterstützen}

\author{
Die topische Therapie von Onychomykosen hat mit einigen Schwierigkeiten \\ zu kämpfen. Womöglich hilft die unterstützende Behandlung mit einem \\ Laser, sie zu überwinden.
}

$\mathrm{D}$ ie lokale Behandlung von pilzbefallenen Finger- oder Zehennägeln kann auf ein Arsenal wirksamer Substanzen zurückgreifen. Die Wirksamkeit wird jedoch nicht selten eingeschränkt, weil es den Präparaten nicht gelingt, die Nageplatte zu durchdringen. Die systemische Therapie ist eine Alternative, $\mathrm{Ne}$ benwirkungen wie etwa toxische Effekte an der Leber setzen ihr freilich Grenzen. Für manche Patienten kommt sie daher nicht infrage. Ein weiteres Problem der Behandlung verpilzter Nägel ist die hohe Rate an Rückfällen.

Dermatologen aus China haben nun untersucht, ob der Einsatz eines Lasers der lokalen Onychomykosetherapie größeren Nachdruck verleihen kann. Beteiligt waren 75 Patienten mit insgesamt 356 befallenen Nägeln. Die Patienten bekamen Terbinafin zur dreimonatigen täglichen lokalen Anwendung verordnet. Zusätzlich wurden die befallenen Nägel dreimal in vierwöchigem Abstand einer fraktionierten Behandlung mit einem $\mathrm{CO}_{2}$-Laser unterzogen.

Nach drei Monaten fiel die Pilzprüfung im Kalilaugenpräparat bei $95 \%$ der Patienten negativ aus, in $92 \%$ der Kulturen waren ebenfalls keine Pilze nachweisbar. Die Quoten fielen allerdings mit der Zeit und lagen nach sechs Monaten noch bei $84 \%$ und $80 \%$.

Die Autoren berechneten die Effektivität der kombinierten Therapie mit $80 \%$. Der Wert lag in etwa gleichauf mit oder sogar über den in früheren Studien für die topische (46\%), orale ( $76 \%$ ), orale plus topische Behandlung ( $86 \%$ ) sowie die Lasertherapie $(70 \%)$ ermittelten Quoten.

Die Erfolgsrate hing auch von der Art der Onychomykose ab, wie ein Vergleich der beiden häufigsten Formen zeigte. Ein vollständiges klinisches Ansprechen auf die kombinierte Therapie aus Terbinafin und Laser zeigten nach sechs Monaten 17 der 36 Patienten mit distaler lateraler subungualer Onychomykose und zwei der 27 Patienten mit totaler dystrophischer Onychomykose. Weitere 13 respektive elf zeigten eine signifikante Response mit einer Verbesserung von mehr als $60 \%$. Entsprechend unterschied sich die Zufriedenheit: Sehr zufrieden mit dem Behandlungsergebnis äußerten sich 27 Patienten mit distaler subungualer und 12 mit totaler dystrophischer Onychomykose.

Fazit: Eine Kombination von Terbinafin und Laser bei Onychomykose scheint Erfolg versprechend. Für die Bewertung der Ergebnisse ist den chinesischen Dermatologen zufolge zweierlei zu berücksichtigen: Es gab keine Kontrollgruppe und die Nachbeobachtungszeit war relativ kurz.

Dr. Robert Bublak

Bhatta AK et al. Fractional carbon-dioxide $\left(\mathrm{CO}_{2}\right)$ laser-assisted topical therapy for the treatment of onychomycosis. J Am Acad Dermatol 2016; 74: 916-23

\title{
Kopfhautnekrosen bei Riesenzellarteriitis
}

Ein 74-jähriger Mann stellte sich wegen seit drei Monaten bestehender Kopfschmerzen, Kieferschmerzen beim Kauen und ausgedehnter Ulzerationen im Bereich der Kopfhaut beim Arzt vor. Eine Visusminderung oder Muskelschmerzen im Sinne einer Polymyalgia rheumatica wurden nicht berichtet. An der Kopfhaut fanden sich ausgedehnte Nekrosen (Abb. A). Beide Temporalarterien waren nicht schmerzhaft, aber kaum tastbar. Die BSG betrug $64 \mathrm{~mm} / \mathrm{h}$, der CRP-Wert $45 \mathrm{mg} / \mathrm{l}$. Unter der Diagnose einer Kopfhautnekrose als Komplikation einer Riesenzellarteriitis wurde der Mann mit 60 mg/d Prednisolon behandelt.

Im Verlauf der folgenden Monate ging die Symptomatik zurück. Auch die Kopfhautläsionen heilten unter tiefer Narbenbildung langsam ab (Abb. B, C). Die Prednisolon-Dosis wurde alle vier Wochen um $10 \mathrm{mg}$ reduziert, bis eine Erhaltungsdosis von 20 $\mathrm{mg} / \mathrm{d}$ erreicht war. Anschließend erfolgte ein langsames Ausschleichen der Dosis.

Kopfhautnekrosen gehören zu den seltenen und potenziell lebensbedrohlichen Komplikationen einer Riesenzellarteriitis. Eine frühzeitige Diagnosestellung und Behandlung sind äußerst wichtig.

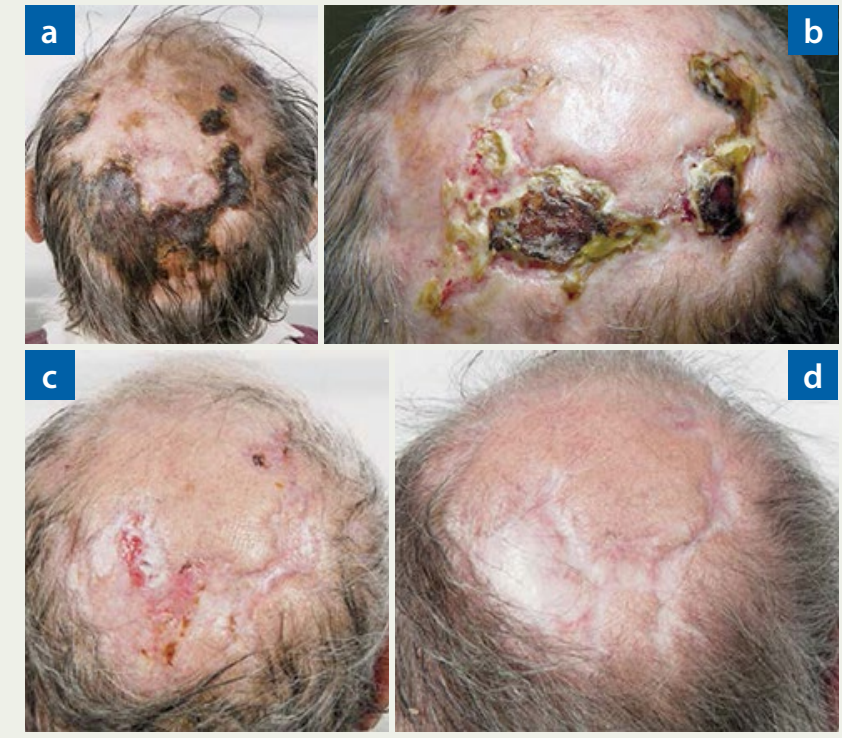

Ausgedehnte Ulzerationen im Bereich der Kopfhaut (A), Abheilung unter tiefer Narbenbildung $(B, C)$, komplette Remission (D). 